\title{
MICROBIOTA INTESTINAL: ROL EN OBESIDAD
}

\section{GUT MICROBIOTA: ROLE IN OBESITY}

\author{
María Magdalena Farías N. (1), Catalina Silva B. (2), Jaime Rozowski N. (2)
}

(1) Escuela de Medicina.

Pontificia Universidad Católica de Chile, Santiago, Chile.

(2) Departamento de Nutrición y Metabolismo.

Pontificia Universidad Católica de Chile, Santiago, Chile.

\begin{abstract}
Obesity and diabetes have become major public health issues in our country. The increasing prevalence of obesity and its association with diabetes might not only be explained by genetic contribution, nutritional habits or decreased physical activity. In addition, recent studies have focused on the intestinal microbiota as environmental factors that increase energy yield from diet, regulate synthesis of gut hormones, peripheral metabolism and systemic inflammatory tone. Obesity is associated with substantial changes in composition of gut microbiota, suggesting that the development of obesity on some people may be due to these changes. Existing evidence warrants further investigation of the microbial ecology oh the human gut and points to modification of the gut microbiota, by functional foods or antibiotics, as one means to treat and prevent obesity.
\end{abstract}

Key words: obesity, diabetes, gut microbiota, probiotics.

Este trabajo fue recibido el 5 de Octubre de 2010 y aceptado para ser publicado el 25 de Mayo de 2011.

\section{INTRODUCCIÓN}

La obesidad se define como un aumento de la grasa corporal en proporción a la altura del paciente. El diagnóstico se establece con la medición del IMC (Índice de Masa Corporal: peso en kilogramos dividido por altura en metros al cuadrado). Un IMC entre 25 y $29,9 \mathrm{~kg} / \mathrm{m}^{2}$ determina el diagnóstico de sobrepeso, mientras que un valor mayor o igual a $30 \mathrm{~kg} / \mathrm{m}^{2}$ se considera como obesidad (1).

Tanto la prevalencia del sobrepeso como de la obesidad han aumentado considerablemente los últimos 10 años tanto en nuestro país como a nivel mundial. En Chile, la prevalencia de sobrepeso y obesidad en población adulta alcanza cifras de hasta $38 \%$ y $22 \%$ respectivamente (2).

El peso corporal de una persona está determinado por una compleja interacción entre el componente genético, ambiental, cultural, social y el gasto energético, el cual está altamente influenciado por el nivel de actividad física de cada individuo (3).

La obesidad ha pasado a ser un importante problema de salud pública en nuestro país (2), por su alta prevalencia y estrecha relación con el desarrollo de ciertas pato- logías tales como diabetes tipo 2, hipertensión arterial, dislipidemia, enfermedades cardiovasculares y cáncer, entre otras (4). Sin embargo, a pesar que la relación entre obesidad y diversas patologías ha sido altamente estudiada, aún existen factores que no se han podido dilucidar completamente. Es por esto que se hace cada vez más relevante el estudio de la obesidad en todos sus aspectos: sus causas, relaciones y tratamientos, además de ampliar los conocimientos hasta ahora descritos.

Recientemente, han surgido múltiples publicaciones que plantean un posible rol de la microbiota intestinal, tanto en el desarrollo de obesidad como de la diabetes. Esta hipótesis nace de la observación de que pacientes obesos presentan una microbiota intestinal distinta a la de individuos normopeso (5), y se explica dado que esta alteración de la microbiota tendría repercusiones en la extracción energética de los alimentos, el metabolismo de ácidos grasos, la síntesis de hormonas intestinales, involucradas en la homeostasis energética; y la regulación de los depósitos corporales de tejido adiposo (6).

Aparentemente, en épocas pasadas en que los humanos debían cazar sus alimentos y pasaban días 
de ayuno, el hecho de presentar una microbiota intestinal que permitiera extraer al máximo la energía de los alimentos y favorecer el almacenamiento de grasa corporal, habría representado una ventaja selectiva, constituyendo un factor más del fenotipo ahorrador. El ser humano probablemente ha ido seleccionando a lo largo de la evolución microorganismos con estas propiedades. Sin embargo, los tiempos han cambiado, y este modelo ahorrador nos juega en contra en la actualidad; ya que tanto los hábitos nutricionales como los tipos de alimentos no son los mismos, por lo que el fenotipo ahorrador de microbiota intestinal, más que una ventaja, pasa a ser considerado como desventaja, contribuyendo a la acumulación de grasa y al consecuente desarrollo de diversas patologías (7).

\section{MICROBIOTA INTESTINAL}

Hasta hace pocos años no existía un amplio conocimiento de la composición de la flora intestinal ni de su rol metabólico en el organismo. Durante la última década, el conocimiento y utilización del ARN ribosomal $16 \mathrm{~S}$ de las bacterias, ha sido de gran utilidad para identificar la gran mayoría de los componentes de la microflora (8).

El intestino humano contiene una gran variedad de microorganismos, dentro de los cuales las bacterias son las que se encuentran en mayor cantidad y diversidad. Las principales bacterias corresponden a tres grandes familias: Firmicutes (gram-positivos), Bacteroidetes (gram-negativos) y Actinobacterias (gram-positivos). Las Firmicutes son la familia que se encuentra en mayor proporción, incluye más de 200 géneros y los más importantes son los Micoplasma, Bacillus y Clostridium (9).

Cada persona presenta una microbiota intestinal única con una gran variabilidad en su composición entre distintos individuos. Al momento de nacer, el intestino es estéril y se coloniza completamente durante el primer año de vida. Existen diversos factores que van modulando la composición de la microbiota a lo largo de la vida. El tipo de parto y la lactancia materna juegan un rol importante en la estabilización de la microflora (10). Posteriormente, es influenciada por la contribución genética, la dieta, y factores ambientales dentro de los cuales destaca el uso de antibióticos. Sin embargo, aún existen factores que contribuirían a la diversidad de la microbiota en la población que no han sido aclarados (8).

\section{MICROBIOTA Y METABOLISMO}

La microbiota intestinal juega un rol importante en la mantención de la función del intestino, ya que estimula su desarrollo, mantiene el recambio epitelial, modula la respuesta inmunológica y participa el metabolismo de algunos medicamentos.
Desde el punto de vista nutricional, las bacterias del intestino juegan un rol crucial ya que participan en la depuración de toxinas provenientes de la dieta; síntesis de micronutrientes como vitamina $\mathrm{K}$, vitamina B12 y ácido fólico; fermentación de sustancias indigeribles; absorción de electrolitos y minerales; y producción de ácidos grasos de cadena corta, los que estimulan el crecimiento y desarrollo de los enterocitos y colonocitos (9).

Con respecto al metabolismo energético, se plantea que la composición de la flora intestinal en un individuo puede determinar una mayor o menor eficacia en la extracción de la energía de la dieta así como una mayor o menor tendencia a depositar el exceso de energía como tejido adiposo (11).

\section{MICROBIOTA, OBESIDAD Y DIABETES}

La asociación entre composición de la flora intestinal y el desarrollo de obesidad nace de observaciones de estudios en ratones germ-free (cuyo intestino es estéril) versus ratones normales. En una serie de experimentos, Backhed et al (12). compararon distintos parámetros entre estos dos grupos de ratones. Pudieron observar que los ratones normales presentaron un $47 \%$ más de tejido adiposo, que los ratones germ-free, ajustadas por ingesta calórica. Posteriormente, realizaron un trasplante de deposiciones desde un ratón normal hacia uno germ-free, observando un aumento de un $60 \%$ del tejido adiposo en el ratón germ free, junto con esto el ratón desarrolló resistencia a la insulina y aumento de niveles de leptina y glucosa circulante.

Otros estudios entre ratones obesos deficientes de leptina (ob/ob) y ratones normo peso $(\mathrm{ob} /+\mathrm{y}+/+)$ fueron concluyentes al observar una distinta composición del la microbiota intestinal. El ratón ob/ob presentó bacterias con mayor expresión genética de enzimas que participan en la extracción de nutrientes, mayor fermentación a nivel intestinal y disminución de las calorías residuales en las deposiciones. También observaron que al trasplantar deposiciones desde ratones ob/ob hacia ratones normopeso, éstos últimos desarrollaban obesidad en un plazo de dos semanas (13). Más tarde, se evidenció cambios en la composición de la microbiota antes y después del desarrollo de diabetes tipo 2 en una población de ratas (14).

En búsqueda de extrapolar estas observaciones a humanos, se han desarrollado distintos estudios con pacientes. Si bien se ha demostrado que distintas patologías se asocian con ciertos perfiles de composición de microbiota, no se ha logrado establecer si estas variantes intestinales juegan un rol de causalidad o corresponden más bien a un efecto de los cambios en la función intestinal explicados por la patología de cada individuo (15).

Se ha podido establecer que los pacientes obesos 
difieren en la composición de sus bacterias intestinales a nivel de Clases. Ya que estos pacientes presentan una mayor proporción de bacterias gram-negativas, miembros del "phylo" Bacteroidetes y a menor proporción de Firmicutes (gram-positivos), es decir un aumento de la relación Firmicutes/Bacteroidetes (F/B) (16). En concordancia con esto, se ha demostrado que los pacientes diabéticos tipo 2 presentan mayor proporción $\mathrm{F} / \mathrm{B}$ y ésta proporción es mayor a medida que la enfermedad y el control glicémico se deteriora (17). El aumento de la relación $\mathrm{F} / \mathrm{B}$ es importante al considerar que el principal componente de las membranas celulares de las bacterias gram-negativos es el lipopolisacárido (LPS), conocido estimulador de la inflamación. Consecuentemente el aumento de LPS contribuye a la resistencia insulínica y por ende, favorece al desarrollo de la diabetes (17). A continuación detallaremos los mecanismos involucrados en la relación de la microbiota intestinal y el metabolismo energético.

\section{Extracción de la energía de la dieta}

El efecto metabólico de la microbiota en relación con la extracción de la energía se entiende gracias al rol que juegan estas bacterias al transformar nutrientes complejos como fibra dietética y mucina en azúcares simples y ácidos grasos de cadena corta. Sin el efecto de la microbiota intestinal tanto la fibra dietética como la mucina serían eliminadas en las deposiciones (18).

Estudios en ratones han demostrado que aquellos que carecen de microbiota intestinal (ratones axénicos) requieren aproximadamente un $30 \%$ más de alimento que los ratones convencionales (colonizados con microbiota intestinal), para lograr crecimientos similares en ambos grupos (12). El mismo efecto se ha constatado en humanos, en quienes el metabolismo bacteriano contribuye a optimizar la extracción calórica de los alimentos hasta en un $10 \%$ (19).

Por otra parte, se ha observado que el proceso de colonización del intestino además de mejorar la extracción energética de los alimentos, aumenta la capacidad de almacenamiento de esta energía en adipocitos, mediante la modificación en la expresión de ciertos genes que influyen en el metabolismo tanto de lípidos como de glúcidos. Esto se ha estudiado principalmente en ratones en los que se ha visto, por ejemplo, mayor expresión de transportadores de monosacáridos en ratones monocolonizados por Bacteroides thetaiotaomicron, los cuales inducen la actividad de la fucosyl-transferasa por los colonocitos con lo que aumenta la absorción de monosacáridos y ácidos grasos de cadena corta, los cuales son utilizados a nivel hepático para sintetizar lípidos de novo (20). En animales con intestino libre de gérmenes, la adquisición de una microbiota intestinal produce aumento de factores de transcripción ChBREP y SREBP-1 así como aumento de las enzimas acetil-CoA carboxilasa y sintasa de ácidos grasos lo que favorece una mayor síntesis de ácidos grasos (12).

El efecto de aumentar el depósito de energía en forma de tejido adiposo se ha podido estudiar en animales. Se ha demostrado que una dieta rica en azúcar y grasa se asocia a niveles menores de FIAF circulante (fasting-induced adipose factor), lo que produce menor control de almacenamiento de grasa en adipocitos y menor lipólisis (ya que al inhibir la expresión intestinal de FIAF, se pierde el efecto inhibidor de la LPL producido por FIAF) (12).

En paralelo, a nivel molecular se ha observado, también en animales, que un intestino libre de bacterias se asocia a una mayor expresión de genes del receptor activado por proliferadores peroxisomales (PPAR \&), el cual presenta conocida actividad catabólica al aumentar la oxidación de ácidos grasos a nivel mitocondrial; así como mayor activación de Adenosin-monofospatoprotein-kinasa enzima activadora del metabolismo lipídico (9).

\section{Inflamación crónica}

La microbiota intestinal regula en gran medida la inmunidad innata y adaptativa, e influye en las respuestas locales y sistémicas; por tanto, también podría influir en la inflamación crónica asociada a la obesidad y resistencia insulínica. Los receptores de reconocimiento celular de las células del sistema inmune innato, como los receptores Toll-like (TLR), constituyen un punto de partida de la inmunidad, que se activa en respuesta a estímulos microbianos o derivados de la dieta (proteínas o lípidos) e informa a las células del sistema inmune para que respondan adecuadamente a estos. Tras su activación por un ligando, los TLR interactúan con diferentes proteínas que activan la transcripción de distintos factores (tales como MAPKs y NF-kB), y la síntesis de diferentes citoquinas y mediadores inmunológicos de la inflamación (21).

En este sentido, se ha demostrado que el lipopolisacárido (LPS) de la membrana celular de las bacterias gram-negativas, como los ácidos grasos saturados de la dieta, pueden actuar como ligando del receptor toll-like 4 (TLR4) y receptor toll-like 2 (TRL2), los cuales tienen la función de estimular la liberación de citoquinas inflamatorias endógenas como el factor de necrosis tumoral (TNF-a), interleuquina 6 (IL-6) y otras citoquinas proinflamatorias relacionadas con la inducción de resistencia a la insulina (22).

En los estudios de Cani et al (23), en modelos ani- 
males sometidos a una dieta rica en grasa la endotoxemia metabólica caracterizada por aumento de los niveles séricos de LPS puede ser un factor inflamatorio causante del aumento de peso corporal, la resistencia insulínica y la diabetes, De hecho, la administración de LPS por vía subcutánea en ratones, produce un aumento de peso y desarrollo de resistencia insulínica. Con lo que es posible plantear que la endotoxemia producida por altos niveles de LPS, en respuesta a niveles elevados de lípidos en la dieta, podría contribuir al desarrollo de resistencia insulínica y diabetes (24).

\section{Hormonas intestinales y efecto incretina}

Una de las formas que tiene el intestino de comunicarse con el hipotálamo, además del sistema nervioso, es mediante la secreción de hormonas que controlan el balance energético (25). Son muchos los neurotransmisores y hormonas involucrados en este proceso. Existe un grupo en especial llamadas incretinas que son producidas por células enteroendocrinas distribuidas a lo largo del tubo digestivo, desde el estómago hasta el colon distal. Aun cuando solo constituyen el 1\% de las células intestinales, el intestino es considerado como un órgano endocrino mayor (26).

Las incretinas (intestinal secretion of insulin) potencian la secreción de insulina en respuesta a la glicemia y son responsables de alrededor del $70 \%$ del nivel de insulina postprandial. Las dos más importantes son el péptido inhibitorio gástrico (GIP) y el péptido similar al glucagón tipo 1 (GLP1) (27).

Se sabe que los productos de fermentación producidos por prebióticos estimulan la diferenciación de las células enteroendocrinas en el epitelio a nivel colónico y la liberación de hormonas digestivas de tipo incretinas las cuales ayudan a regular los niveles de glicemia (7).

\section{POTENCIALES INTERVENCIONES}

El mejor tratamiento no quirúrgico para la obesidad corresponde a cambios de estilo de vida, alimentación saludable y ejercicio físico, para lograr un óptimo funcionamiento biológico de las reservas energéticas y prevenir un balance energético positivo (3). En función de la relación establecida entre la obesidad, el metabolismo, la inmunidad y la microbiota intestinal, el uso de estrategias dietéticas destinadas a modular la composición de la microbiota se ha propuesto como medio para controlar los trastornos metabólicos de forma más eficaz. La mayoría de los estudios al respecto se basan en la administración de probióticos y prebióticos.

Un probiótico es una preparación o producto que contiene microorganismos definidos, viables y en cantidades suficientes, que modulan la microflora en uno de los compartimentos del huésped y ejercen efectos beneficios sobre la salud. Estos microorganismos no colonizan de forma permanente el intestino y tienen la capacidad de mantenerse vivos a lo largo de todo el trayecto del tubo digestivo, es decir son resistentes a la acidez gástrica, enzimas intestinales y sales biliares (28). El uso de probióticos ha sido ampliamente estudiado en cuadros de gastroenteritis aguda, enterocolitis necrotizante, enfermedad inflamatoria intestinal, síndrome de intestino irritable, y constipación entre otras (28).

Recientemente, se ha demostrado que el consumo de probióticos se asocia a una mayor concentración de bacterias gram-positivas y a una disminución de las gram-negativas en las deposiciones, con la consiguiente disminución de los niveles de LPS circulante, lo cual podría disminuir el desarrollo de la endotoxemia, y por ende, el desarrollo de obesidad y resistencia insulínica (29). Un estudio en ratas demostró que la administración de leche fermentada con Lactobacillus gasseri SBT2055 durante 4 semanas, produjo una reducción en el tamaño, así como un aumento del número de los adipositos del tejido adiposo retroperitoneal y mesentérico, además de reducir los niveles séricos de leptina (30). Otro estudio en ratones demostró que la administración de una preparación con diversas cepas de probióticos (Lactobacilus, Bifidobacterium y Streptocossus) mejoró la esteatosis y resistencia a la insulina inducida por una dieta rica en grasa (31).

Los prebióticos, en cambio, corresponden a oligosacáridos indigeribles en la parte alta del tubo digestivo. La presencia de oligosacaridos estimula el crecimiento y la actividad metabólica de las bacterias beneficiosas, ya que los pueden usar como substratos y fermentarlos. Los más conocidos son la inulina y la oligofructosa, los cuales también ejercen efectos beneficiosos sobre la ingesta y diversos parámetros metabólicos $(32,33)$, lo que se explica, en parte, gracias a que el aumento de las bacterias gram-positivos se correlaciona con una disminución del LPS, y por ende, se normaliza el tono inflamatorio por reducción de la endotoxemia (34).

En modelos animales, los efectos beneficiosos de la oligofructosa se han vinculado a los productos de su fermentación, responsables de la generación de ácidos grasos de cadena corta como el ácido butírico. Éste promueve la diferenciación de células L en el colon proximal y aumenta así la síntesis de GLP-1, lo que favorece la sensación de saciedad (35). Esto se estudió en ratas, las cuales presentaron niveles aumentados de GLP-1 así como un efecto protector frente a la ganancia de peso al adicionarles oligofructosa en la dieta (36). También se ha descrito el efecto en otros péptidos gastrointestinales 
(como el PYY y la grelina) (29).

Diversos estudios similares sugieren el rol de los probióticos y prebióticos en la regulación del crecimiento del tejido adiposo, inflamación sistémica, saciedad frente a la alimentación y posiblemente, en obesidad, sin embargo faltan estudios que demuestren un efecto terapéutico real.

\section{COMENTARIO}

En las últimas décadas, se han logrado avances significativos en la comprensión de los diversos mecanismos involucrados en el desarrollo de la obesidad. La evidencia científica se inclina a proponer que la obesidad corresponde al resultado de la interacción de múltiples factores.

Recientemente, la aplicación de técnicas científicas altamente específicas, así como estudios en animales colonizados selectivamente por ciertas bacterias, han permitido establecer asociaciones bastante específicas ente la inmunidad, la inflamación y el metabolismo energético. Por lo que se ha propuesto que la composición de la microbiota intestinal constituye uno de los factores involucrados en el desarrollo de la obesidad.

Extensos estudios epidemiológicos serán necesarios para confirmar si realmente la obesidad se encuentra asociada, causalmente o no, con una modificación de la microbiota intestinal en seres humanos.

\section{RESUMEN}

La obesidad es una patología metabólica altamente prevalente en nuestro país y constituye un gran problema de salud pública. Según lo han demostrado recientes estudios, la obesidad y su asociación con la diabetes no son únicamente el resultado de la contribución genética, los hábitos alimentarios o la falta de actividad física, sino que también se ha comprobado que la microbiota intestinal constituye un factor ambiental determinante en el desarrollo de estas patologías. Este vínculo estaría relacionado con una modulación, por parte de la microbiota, en la extracción energética de los alimentos, la secreción de hormonas intestinales, el metabolismo a nivel periférico y el tono inflamatorio sistémico. Lo anterior se refleja en las diferencias significativas en cuanto a la composición de la microbiota intestinal que se observa en quienes padecen obesidad. En relación a la evidencia actual, resulta importante investigar los efectos en la modificación de la microbiota intestinal, mediante el uso alimentos funcionales y antibióticos, a fin de aplicar tanto tratamientos terapéuticos como preventivos contra el desarrollo de la obesidad.

Palabras clave: obesidad, diabetes, microbiota intestinal, probióticos.
Dirigir la correspondencia a:

María Magdalena Farías N

Albacete 4420, Depto. 56

Las Condes - Santiago, Chile

Teléfono. 99945244

E-mail:mmfarias@gmail.com

\section{BIBLIOGRAFÍA}

1. Kim S, Popkin BM. Commentary: understanding the epidemiology of overweight and obesity: a real global public health concern. Int J Epidemiol 2006;35:60-7.

2. Ministerio de Salud/Instituto Nacional de Estadística. Encuesta Nacional de Salud 2003.

3. Farias MM, Cuevas AM, Rodriguez F. Set-point theory and obesity. Metab Syndr Relat Disor 2011;9:85-9.

4. Lewis C, McTigue K, Burke L, Poirier P, Eckel R, Howard B, et al. Mortality, health outcomes, and body mass index in the overweight range: a science advisory from the American Heart Association. Circulation 2009;119:3263-71.

5. Tilg H. Obesity, metabolic syndrome, and microbiota: multiple interactions. J Clin Gastroenterol 2010;44 S1:S16-8.

6. Turnbaugh PJ, Ley RE, Mahowald MA, Magrini V, Mardis ER, Gordon JI. An obesity-associated gut microbiome with increased capacity for energy harvest. Nature 2006; 444:1027-31.

7. Morales P, Brignardello J, Gotteland M. La microbiota intestinal: Un nuevo actor en el desarrollo de la obesidad. Rev Med Chil 2010;138:1020-7.

8. Hattori M, Taylor TD. The human intestinal mirobiome: A new frontier of human biology. DNA Res 2009;16;1-12.

9. DiBaise JK, Zhang H, Crowell MD, KrajmalnikBrown R, Decker GA, Rittmann BE, et al. Gut microbiota and its possible relationship with obesity. Mayo Clin Proc 2008;83:460-9.

10. Martin R, Langa S, Reviriego C, Jimínez E, Marín ML, Xaus J, et al. Human milk is a sorce of lactic bacteria fot the infant gut. J Pediatr 2003;143:754-8.

11. Mai V, McCrary QM, Sinha R, Glei M. Associations between dietary habits and body mass index with gut microbiota composition and fecal water genotoxicity: an observational study in African American and Caucasian American volunteers. Nutr J 2009;8:49.

12. Bäckhed F, Ding H, Wang T, Hooper LV, Koh GY, Nagy A, et al. The gut microbiota as an environmental factor that regulates fat storage. Proc Natl Acad Sci USA 2004; 101:15718-23. 
13. Ley RE, Bäckhed F, Turnbaugh P, Lozupone CA, Knight RD, Gordon JI. Obesity alters gut microbial ecology. Proc Natl Acad Sci USA 2005;102:110705.

14. Cani PD, Bibiloni, Knauf C, Eaget A, Neyrinck A, Delzenne N, et al. Changes in gut microbiota control metabolic endotoxinemia-induced inflammation in high-fat diet-induced obesity and diabetes in mice. Diabetes 2008;57:1470-81.

15. Mai V, Draganov PV. Recent advances and remaining gaps in our knowledge of associations between gut microbiota and human health. World J Gastro-

16.

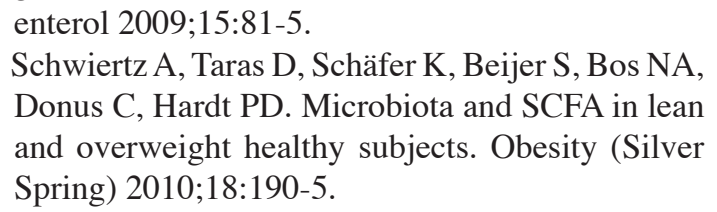

17. Larsen N, Vogensen F, van den Berg F, Nielsen D, Andreasen A, Pedersen B. Gut Microbiota in human adults with type 2 diabetes differs from non-diabetic adults. PLos ONE 2010;5:e9085.

18. Vrieze A, Holleman F, Zoetendal G, de Vos WM, Hoesktra JB, Nieuwdorp M. The environment within: how gut microbiota may influence metabolism and body composition. Diabetologia 2001; 53:606-13.

19. Ley RE, Turnbaugh PJ, Klein S, Gordon JI. Microbial ecology: human gut microbes associated with obesity. Nature 2006;444:1022-3.

20. Hooper LV, Wong MH, Thelin A, Hansson L, Falk PG, Gordon JI. Molecular analysis of commensal host-microbial relationships in the intestine. Science 2001;291:881-4.

21. Sanz Y, Santacruz A, Dalmau J. Influencia de la microbiota intestinal en la obesidad y las alteraciones del metabolismo. Acta Pediatr Esp 2009;67:437-42

22. Ley RE. Obesity and the human microbiome. Curr Opin Gastroenterol 2010;26:5-11.

23. Cani PD, Amar J, Iglesias MA, Poggi M, Knauf C, Bastelica D, et al. Metabolic endotoxaemia initiates obesity and insulin resistance. Diabetes 2007;56:1761-72.

24. Amar J, Burcelin R, Ruidavets J, Cani P, Fauvel J, Alessi M, et al. Energy intake is associated with endotoxemia in apparently healthy men. Am J Clin
Nutr 2008; 87: 1219-23.

25. Druce MR, Small CJ, Bloom SR. Minireview: Gut peptides regulating satiety. Endocrinol 2004; 145:2660-5.

26. Drucker DJ. Biological actions and therapeutic potential of the glucagon-like peptides. Gastroenterol 2002; 122:531-44.

27. Cani PD, Possemiers S, Van de Wiele T, Guiaot Y, Everard A, Rottier O, et al. Changes in gut microbiota control inflammation in obese mice through a mechanism involving GLP-2-driven improvement of gut permeability. Gut 2009;58:1091-103.

28. Schrezenmeir J, Vrese M. Probiotics, prebiotics, and synbiotics-approaching a definition. Am J Clin Nutr 2001;73:361S-4S.

29. Cani PD, Neyrinck AM, Fava F, et al. Selective increases of bifidobacteria in gut microflora improve high-fat-diet-induced diabetes in mice through a mechanism associated with endotoxaemia. Diabetologia 2007;50:2374-83.

30. Sato M, Uzu K, Yoshida T, Hamad EM, Kawakami $\mathrm{H}$, Matsuyama H, Abd El-Gawad IA, Imaizumi K. Effects of milk fermented by Lactobacillus gasseri SBT2055 on adipocyte size in rats. Br J Nutr 2008;99:1013-7.

31. Ma X, Hua J, Li Z. Probiotics improve high fat diet-induced hepatic steatosis and insulin resistance by increasing hepatic NKT cells. J Hepatol 2008 ;49:821-30.

32. Gibson G. R., Roberfroid M. B. Dietary modulation of the human colonic microbiota: introducing the concept of prebiotics. J. Nutr 1995;125:1401-12.

33. Gibson G. Dietary Modulation of the Human Gut Microflora Using the Prebiotics Oligofructose and Inulin. J Nutr 1999;129:1438S-41S.

34. Jenkins D, Kendall C, Vuksan V. Inulin, oligofructose, and intestinal function. J Nutr 1999; 129:1431S-33S.

35. Delzenne N, Cani PD, Daubioul C, Neyrinck A. Impact of insulin and oligofructose on gastrointestinal peptides. Br J Nutr 2005;93:S157-61.

36. Cani PD, Dewever C, Delzenne NM. Inulin-type fructans modulate gastrointestinal peptides involved in appetite regulation (glucagons-like peptide-1 and ghrelin) in rats. Br J Nutr 2004;92:521-6. 